\title{
DIMENSIONAL ACCURACY BETWEEN THREE DIFFERENT INDIRECT SCANNERS FOR CAD/CAM SYSTEMS - AN IN-VITRO STUDY
}

\author{
Mohamed A. Elbasha*, Ahmed M.Y. El-Kouedi**, Moemen A. Abdelkader***
}

\begin{abstract}
Objective: Extraoral scanning is considered an alternative for conventional work flow of crown and bridge framework. The purpose of this study is to compare the accuracy of three different indirect scanners (Sirona Ineos x5, 3 shape D800, D of Freedom hd) for CAD/CAM systems with different arrangement of restored dentition(single crown, multiple crowns, simple bridge). The hypotheses was that different arrangement of restored dentition will affect the accuracy of different scanning systems.
\end{abstract}

Materials and methods: In this study, three different arrangement of restored dentition (single crown, simple bridge and multiple crowns) were scanned by CMM and three different extraoral scanners (Dof, 3 Shape and Sironoa) to get 45 STL files. These STL files were compared to measure accuracy (trueness and precision) by an analyzed specific software (poly works).

Results: The analysis revealed statistically non- significant differences in precision and trueness between the three different scanners.

Conclusion: Within the limitations of this study, the following recommendations can be made: The analysis revealed statistically a non- significant difference in precision and trueness between the three different scanners.

KEY WORDS: CAD/CAM, Trueness, Precision, Indirect Scanners

\section{INTRODUCTION}

Computer-aided design/computer aided manufacturing (CAD/CAM) technology has brought a whole new range of techniques and materials to dentistry. This technology has evolved since its introduction in the 1980s and is now part of daily practice $^{(1,2)}$.

The CAD/CAM systems produce higher quality restorations by using industrially prepared materials and a standardized manufacturing process ${ }^{(3)}$. The workflow consists of 3 main steps: surface scanning, restoration design, and manufacture ${ }^{(4)}$.

Two main types of dental CAD/CAM scanners exist: intraoral scanners are used Chairside to scan patients' dental arches; extraoral scanners are used in the dental laboratory to scan casts ${ }^{(5)}$. Both types of scanners develop a digital model of the patient's mouth using CAD software. At the end of the process, 3D printers or milling machines that use CAM technology fabricate the definitive physical model or restoration using ceramics, polymers, metals, or other materials ${ }^{(6,7)}$.

Although one of the objectives of computeraided technology is to increase the accuracy of the manufacturing process ${ }^{(\mathbf{8})}$ few studies have analyzed the effect of scanning systems on the accuracy of fit, which is one of most important criteria for the long-term success of restorations ${ }^{(9)}$. This digital work flow process and the accuracy of the scanning

\footnotetext{
* B.D.S 2009 G, Faculty of Dental Medicine MUST University,

** Assistant Professor, Department of Crown and Bridge, Faculty of Dental Medicine, Boys, Cairo, Al-Azhar University

${ }^{* * * *}$ Lecturer, Department of Crown and Bridge, Faculty of Dental Medicine, Boys, Cairo, Al-Azhar University
} 
and data acquisition step in the $\mathrm{CAD} / \mathrm{CAM}$ process is of vital importance ${ }^{(10)}$ The purpose of our study was not to evaluate the accuracy of three different indirect scanners (Sirona Ineos x5, 3 shape D800, Dof Freedom hd) for CAD/CAM systems with different arrangement of restored dentition (single crown, multiple crowns, simple bridge).

\section{HYPOTHESIS}

The hypothesis of this study was that different arrangement of restored dentitions will affect the accuracy of different scanning systems.

\section{MATERIALS AND METHODS}

Three different extra oral (two different light scanner and a laser scanner) were used with simulating the clinical situations and conditions as much as possible.

\section{Construction of reference model:}

An articulated acrylic resin typodont" of upper and lower arch was used in this study. The typodont teeth were prepared into three different patterns of restored dentition to mimic clinical situation as follow:

1. Pattern 1: Single crown

2. Pattern 2: Multiple upper anterior crowns (from canine to canine)

3. Pattern 3: Simple fixed-fixed dental prosthesis (missing lower first premolar)

\section{Abutments preparation:}

CNC machine ${ }^{* *}$ was used for preparation of abutments. The CNC router with $1000 \times 600 \mathrm{~mm}$ machining area was used with maximum cutting speed. The Max. Cutting Speed is $8000 \mathrm{~mm} / \mathrm{min}$. The ball Screws / spindles diameter is $12 / 12 \mathrm{~mm}$.
The prepared typodont was duplicated to obtain the reference model. The duplication was done by additional silicone impression material (polyvinyl siloxane $)^{* * *}$. Acrylic resin special tray was constructed with spacer $4-5 \mathrm{~mm}$ to compensate for material thickness. The impression technique used for duplication was two-step impression technique ${ }^{(11)}$

\section{Preparation of the reference model for measure-} ment on Coordinate measuring machine (CMM):

\section{A- Creation of point angles:}

The measurements obtained from CMM machine are linear measurements. These linear measurements require starting and ending points (point angles) with precise definition. In order to define these points angle on each abutment, a ledge was prepared on the incisal or occlusal surface of each abutment. The ledge was prepared by milling surveyor using flat end stone (parallel side). In the first pattern (lower molar) two ledges were performed, one buccal and one lingual while in the second and third patterns one ledge only was prepared on the incisal and occlusal surfaces.

- In pattern one (lower single crown), point angles were performed on the buccal and lingual surfaces and numbered as $5 \& 6$.

- In pattern two (upper multiple crowns) point angles were performed on teeth no $(13,12,11,21,22,23)$ and are numbered from 2 to 7 .

- In pattern three (lower bridge) point angles were placed on teeth no $(31,32,34)$ and numbered from 2 to 4 .

\section{$B$-Creation of reference planes:}

The linear measurements by CMM was compared with the same distances taken by optical scan (virtual $3 \mathrm{~d}$ model scan). In order to accomplish this step,

\footnotetext{
* 500A, Nissin, Japan

** High z-t 1000 -Germany

*** Lascode -Italy
} 
the reference model had a definite reference plane to aid in the alignment and same determination of starting and ending points for the same linear measurements on the optical scan.

The reference planes are three perpendicular planes $(\mathrm{x}, \mathrm{y} \& \mathrm{z})$, these planes were made using milling surveyor on the last molar of each arch.

\section{Obtaining Linear Reference Scan:}

The Reference model was scanned for each of the three groups of the prepared teeth separately with the reference scanner which is a coordinate measuring machine ${ }^{* * * *}$. All measurements were done by linear measurement method where the machine probe moves starting from the reference point around the full arch to measure distances between the incisal edge points.

\section{Optical Scanning Of the reference model:}

The reference model was scanned with 3 different extra oral scanners with different characteristics and properties.

For every test group (each pattern), five scans were done with the three scanners where $(\mathrm{N})$ is the number of the scans thus we get 45 STL files to compare. All data sets from the three dental scanners are exported as STL files and processed into specific software for accuracy measurements.

\section{Data analysis and processing:}

A 3D data analyzing software (Poly works) was used to evaluate and compare the discrepancy among these STL files using best fit algorithm to measure precision while trueness was measured using linear measurements. This software was able to trim all irrelevant area $(0.5 \mathrm{~mm}$ away from the finish line of the three patterns), with a range of tolerance of $0.05 \mathrm{~mm}$.
Each of the three patterns were scanned five times of the three scanners, thus we obtained 45 scans (i.e. $3 * 5 * 3=45$ ).

A) Trueness measurements were done by comparing the reference model linear distances from CMM along with the first STL file of each of the scanned data of the three dental scanners.

B) Precision measurements were done by comparing each scan (5 scans) within the test group where the first scan was taken as a reference scan. The precision measurements were done by color map superimposition.

\section{RESULTS}

The data were tested for normality using the Anderson-Darling test and for homogeneity variances prior to further statistical analysis. The continuous variables described by mean and standard deviation (Mean, SD). Independent-Samples T test and One-way ANOVA were used to compare between continuous variables. A two-tailed $\mathrm{p}<0.05$ was considered statistically significant.

The analysis revealed statistically non- significant differences in precision and trueness between the three different scanners. As we described before, the accuracy of measurements as having two components, trueness and precision; trueness defines the extent to which the measurements deviate from the true size of the object, and precision shows the fluctuation of the measured results.

The results of trueness measurements for pattern 1 showed non-significant difference between the reference model and the three different scanners. The standard deviations were shown in table (1) states best trueness was for 3 shape scanners (p:0.092) followed by Dof scanner and lowest trueness was for Sirona scanner (p:0.0952)

***** Wilcox, hexagon metrology 
TABLE (1) Differences in trueness measurements of the three scanners in pattern 1 distance 3 .

\begin{tabular}{|l|c|c|c|}
\hline & \multicolumn{2}{|c|}{$\begin{array}{c}\text { Pattern1 D3 } \\
\text { (Ref. mean=7.734) }\end{array}$} & \multirow{2}{*}{ P. value } \\
\cline { 2 - 3 } & Mean & Dev. & \\
\hline 3 shape scanners & 7.736 & 0.002 & $\mathbf{0 . 9 9 2}$ \\
\hline Dof scanner & 7.739 & 0.005 & 0.980 \\
\hline Sirona scanner & 7.722 & -0.012 & 0.952 \\
\hline
\end{tabular}

The precision measurements of the three scanners for pattern 1 showed the best values for Sirona scanner followed by Dof scanner and the lowest values was for 3 shape scanners. All values show a non-significant difference between three scanners.

For pattern 2, standard deviations show nonsignificant difference between the three different scanners for distances 1,2 and 3, with the best trueness in distance 1 for 3 shape scanners (p:0.097) followed by Dof scanner (p:0.993) and lowest trueness for Sirona (p:0.841) table 2.

Distance 2 shows the best trueness value for 3 shape scanners (p:0.999) followed by dof scanner (p:0.993), lowest trueness value for Sirona scanner (p:0.973).

Measurements of distance 3 showed the best trueness values for Sirona scanner (p:0.999) followed by dof (p:0.994) and 3 shape with the same value (p:0.994) table 8.

Trueness measurements of pattern 3(bridge) showed non-significant difference in both distance 1 and distance 2 .

In distance 1 best trueness value was for 3 shape scanners (p:0.994) followed by Dof scanner (p:0.988) and lowest values for Sirona (p:0.911) table 3.
TABLE (2) Differences in trueness measurements of the three scanners in pattern 3 distance 1 .

\begin{tabular}{|l|c|c|c|}
\hline \multirow{2}{*}{} & \multicolumn{2}{|c|}{$\begin{array}{c}\text { Pattern3 D1 } \\
\text { (Ref. mean=5.189) }\end{array}$} & \multirow{2}{*}{ P. value } \\
\cline { 2 - 3 } & Mean & Dev. & \\
\hline 3 shape scanners & 5.190 & 0.001 & \multirow{0}{*}{$\mathbf{0 . 9 9 4}$} \\
\hline Dof scanner & 5.191 & 0.002 & 0.988 \\
\hline Sirona scanner & 5.174 & -0.015 & 0.911 \\
\hline
\end{tabular}

Distance 2 showed best trueness value for Sirona (p:0.994) followed by Dof scanner (p:0.983) and lowest value for 3 shape (P:0.991) table 3 .

TABLE (3) Differences in trueness measurements of the three scanners in pattern 3 distance 2.

\begin{tabular}{|l|c|c|c|}
\hline \multirow{2}{*}{} & \multicolumn{2}{|c|}{$\begin{array}{r}\text { Pattern3 D2 (Ref. } \\
\text { mean=15.778) }\end{array}$} & \multirow{2}{*}{ P. value } \\
\cline { 2 - 3 } & Mean & Dev. & \\
\hline 3 shape scanners & 15.773 & -0.005 & 0.991 \\
\hline Dof scanner & 15.787 & 0.009 & 0.983 \\
\hline Sirona scanner & 15.775 & -0.003 & $\mathbf{0 . 9 9 4}$ \\
\hline
\end{tabular}

*: Statistically significant at $p \leq 0.05$

\section{DISCUSSION}

$\mathrm{CAD} / \mathrm{CAM}$ technology has a great participation in digital dentistry as a trend to shift to complete digital work flow ${ }^{(12)}$

The intention is to replace conventional impressions by digital impressions. For this purpose, trueness and precision must be technically at least equivalent to the conventional impression. These first in vitro data shows that it is technically possible to fulfill this requirement with the presentday methods ${ }^{(13)}$

During preparation of the reference model, we used 3 different clinical situations, firstly the common single crown on a molar (pattern 1), 
multiple anterior crowns for a smile make-over situation (Pattern 2), and a fixed partial denture (pattern 3). In pattern 3 (lower bridge) a piece of wax was used to block the space of the missed $\operatorname{tooth}^{(14)}$

Although CMM wasted a lot of time for obtaining the reference scans but it offered a very accurate and precise scan value. The vertical grooves made on the teeth edges were necessary to maintain reference points from where the linear measurements between teeth can be done ${ }^{(15)}$

The choice of the extra oral scanners was done according to their different specifications. (LED blue light (Sirona Ineos x5) white light (dof freedom hd) and 3 shape d800(laser scanner)

Light scanners are more accurate. The overall point space can be controlled at $0.2 \mathrm{~mm} \sim 0.4 \mathrm{~mm}$, improving significantly the resolution by 2 to 5 times. This was showed true in our study as the Sirona Ineos x 5 scanner showed a better result in many situations ${ }^{(16)}$

Trueness measurements results showed the best values for 3 shape scanners related to short distances which was represented by pattern 1 , distance $1 \& 2$ in pattern 2 and distance 1 in pattern 3 .

Best trueness values were found in Sirona scanner in long distances which is represented by distance 3 pattern 2 and distance 2 pattern 3 .

The lowest trueness values for Sirona scanner were found in pattern 2 distances 1 and 2 .

Sirona scanner showed best precision values in pattern 1 points $4,5 \& 7$ in pattern 2 and point 4 in pattern 3 . This can be explained by that the precision of Sirona increases as we go in posterior regions ${ }^{(17)}$

Precision of Sirona scanner showed the lowest values in points 2, 3 in pattern 2 which are the most anterior regions with point 2 in pattern $3^{(18)}$
Our study didn't check the accuracy in relation to different positions on tooth surface like axial walls or occlusal surfaces so future studies need to compare such variable to give better representation of the different scanners available in the market.

\section{CONCLUSION}

Within the limitations of this study, the following recommendations can be made:

The analysis revealed statistically a non- significant difference in precision and trueness between the three different scanners.

\section{REFERENCES}

1. Duret F, Preston JD. CAD/CAM imaging in dentistry. Curr Opin Dent 1991;1: 150-4.

2. Goodacre CJ, Garbacea A, Naylor WP, Daher T, Marchack CB, Lowry J. CAD/ CAM fabricated complete dentures: concepts and clinical methods of obtaining required morphological data. J Prosthet Dent 2012; 107:34-46.

3. Prasad R, Al-Kheraif AA. Three-dimensional accuracy of $\mathrm{CAD} / \mathrm{CAM}$ titanium and ceramic superstructures for implant abutments using spiral scan microtomography. Int $\mathrm{J}$ Prosthodont 2013; 26:451-7.

4. Freedman M, Quinn F, O'Sullivan M. Single unit CAD/ CAM restorations: a literature review. J Ir Dent Assoc 2007; 53:38-45

5. Tamim H, Skjerven H, Ekfeldt A, Ronold HJ. Clinical evaluation of $\mathrm{CAD} / \mathrm{CAM}$ metal-ceramic posterior crowns fabricated from intraoral digital impressions. Int J Prosthodont 2014; 27:331-7.

6. Kachata PR, Geissberger MJ. Dentistry a la carte: in office CAD/CAM technology. J Calif Dent Assoc 2010; 38:323-30

7. Bosch G, Ender A, Mehl A. A 3-dimensional accuracy analysis of chairside $\mathrm{CAD} / \mathrm{CAM}$ milling processes. J Prosthet Dent 2014; 112:1425-31.

8. Chan DC, Chung AK, Haines J, Yau EH, Kuo CC. The accuracy of optical scanning: influence of convergence and die preparation. Oper Dent 2011;36: 486-91.

9. Sachs C, Groesser J, Stadelmann M, Schweiger J, Erdelt K, Beuer F. Full-arch prostheses from translucent zirconia: accuracy of fit. Dent Mater 2014;30: 817-23. 
10. Peumans $\mathrm{M}$ et al. Five-year clinical performance of porcelain veneers. Quintessence Int 1998;29(4):211-21.

11. ceyhan ja, Johnson gh, lepe x. The effect of tray selection, viscosity of impression material, and sequence of pour on the accuracy of dies made from dual-arch impressions Prosthet dent 2013;90:143-149.

12. Persson AS, Oden A, Andersson M, Sandborgh-Englund G. Digitization of simulated clinical dental impressions: virtual three-dimensional analysis of exactness. Dent Mater 2009; $25: 62$

13. Khardekar R, Burton G, McMains S. Finding feasible mold parting directions using graphics hardware. Computaided des 2006; 38: 327-41.
14. Chang M, Park SC. Automated scanning of dental impressions. Comput-aided des J dent 2009; 41:404-11.

15. Quaas S, Rudolph H, Luthardt RG, Direct mechanical data acquisition of dental impressions for the manufacturing of CAD/ CAM restorations. J Dent 2007; 35:903-908.

16. Chmielewski, Sz., Tompalski, P. Estimating outdoor advertising media visibility with voxel-based approach. Applied Geography 2016; 87:1-13.

17. Patzelt SB, Emmanouilidi A, Stampf S, Strub JR, Att W, Accuracy of full-arch scans using intraoral scanners. Clin Oral Investig 2015; 18:1687-1694.

18. Ender A, Mehl A In-vitro evaluation of the accuracy of conventional and digital methods of obtaining full-arch dental impressions Quintessence Int 2007;46:9-17. 